Elsevier Editorial System(tm) for Journal of Alloys and Compounds

Manuscript Draft

Manuscript Number: JALCOM-D-08-01798R1

Title: Additivity of reinforcing mechanisms during creep of metal matrix composites: role of the microstructure and the processing route

Article Type: Normal Paper

Keywords: A. metals; B. powder metallurgy; D. high temperature alloys; D. creep.

Corresponding Author: Dr. Gaspar González-Doncel, PhD

Corresponding Author's Institution: CENIM, C.S.I.C.

First Author: Ricardo Fernández, Phd

Order of Authors: Ricardo Fernández, Phd; Gaspar González-Doncel, PhD

Abstract: During decades, great efforts have been devoted to understand and predict the creep behavior of discontinuously reinforced metal matrix composites, particularly aluminum alloy matrix materials. As a result of all these investigations, however, a confuse panorama of the precise role of the reinforcing particles on the enhanced creep response of these materials rules the present understanding. Here, an analysis of the reasons that have driven to this situation is presented. Also, a simple and generalized framework of the relevant mechanisms required to understand this behavior is made. This view is based on previous work by these authors on 6061 Al alloy, prepared by ingot and powder metallurgy, and 6061 $\mathrm{Al}-15 \mathrm{vol} \% \mathrm{SiCw}$ composite, as well as a data analysis of published investigations aimed at clarifying the above situation. The additivity of the proposed contributions and the potential damage mechanism are discussed in the context of the processing route employed. 


\title{
Additivity of reinforcing mechanisms during creep of metal matrix composites: role of the microstructure and the processing route
}

\author{
Ricardo Fernández ${ }^{\S}$, Gaspar González-Doncel* \\ Dept. of Physical Metallurgy, Centro Nacional de Investigaciones Metalúrgicas (CENIM), \\ C.S.I.C., Av. de Gregorio del Amo 8, E-28040 Madrid, Spain \\ ${ }^{\S}$ Present address, Thin Film R\&D Dept. INDO, SA, C/ Alcalde Barnils 72, 08174 Sant Cugat \\ del Vallés, Barcelona, Spain
}

\begin{abstract}
During decades, great efforts have been devoted to understand and predict the creep behavior of discontinuously reinforced metal matrix composites, particularly aluminum alloy matrix materials. As a result of all these investigations, however, a confuse panorama of the precise role of the reinforcing particles on the enhanced creep response of these materials rules the present understanding. Here, an analysis of the reasons that have driven to this situation is made. Also, a simple and generalized framework of the relevant mechanisms required to understand this behavior is proposed. This view is based on previous work by these authors on 6061 Al alloy, prepared by ingot and powder metallurgy, and $6061 \mathrm{Al}-15 \mathrm{vol} \% \mathrm{SiC}_{\mathrm{w}}$ composite, as well as a data analysis of published investigations aimed at clarifying the above situation. The additivity of the proposed contributions and the potential damage mechanism are discussed in the context of the processing route employed.
\end{abstract}

Key words,

A. metals; B. powder metallurgy; D. high temperature alloys; D. creep.

* Corresponding author, ggd@ cenim.csic.es. Phone, +34 915538900 ext.337 


\section{1.- Introduction}

Metal matrix composites, MMCs, in particular aluminum alloys with discontinuous ceramic reinforcement, such as $\mathrm{SiC}$ or $\mathrm{B}_{4} \mathrm{C}$ particles, are known to improve mechanical properties of monolithic alloys without a significant weight increase [1]. Because of this, they are attractive structural materials in engineering applications in the transportation sector [2]. Although these materials were initially conceived to improve the mechanical properties at room temperature, it was soon encountered that their high temperature behavior also improved with respect the creep response of the un-reinforced alloy. This finding extended further the potential use of these materials such as in engine components and brake rotors in terrestrial and aerospace vehicles $[3,4]$.

Since this finding, tremendous efforts to understand the enhanced creep behavior of MMCs have been carried out by different researchers. Despite these attempts, however, the present capability to understand and predict this behavior is rather weak such that several reasons are commonly invoked to explain it. This confusing picture is, to our understanding, due to the following three reasons:

a) Very different aluminum alloys have been used for composites preparation. Pure aluminium and aluminum alloys such as stable 8009Al alloy or Al-Si alloys (employed in the automotive sector) have been used as the metallic matrix for these composites. In many cases, however, not even the creep behavior of the aluminum matrix alloy is yet fully understood. More specifically, since the initial goal was to develop composites with superior room temperature properties (to increase metallic alloys stiffness), many of them are conventional age hardenable alloys (i.e., which undergo well known precipitation sequences after annealing at moderate temperature from a solid solution condition [5-9]) developed for room temperature applications. In particular, alloys such as those of the $2 \mathrm{xxx}$ and $6 \mathrm{xxx}$ series, have been frequently used. In these cases, when creep tests are carried out at temperatures at which the kinetics of precipitate formation is sufficiently fast, an unavoidable, non-controlled, evolution of this precipitation from the beginning of the creep test will occur. This is a clear difficulty if a rigorous analysis of the data obtained from tests conducted at different temperatures or lasting different times is sought: Dislocation interaction with the precipitates will evolve differently at the different temperaturetime of testing and the mechanical properties will evolve. Surprisingly, these precipitation phenomena are very rarely considered in research papers in the literature [10], but are very important if understanding the creep of age-hardenable aluminum alloys and their respective MMCs is sought. 
b) The processing route employed, specifically ingot metallurgy, IM, vs. powder metallurgy, PM, can be of particular importance for composites creep behavior through the different elements which these procedures introduce in material's microstructure. On one hand, IM requires melting the alloy to introduce and disperse the ceramic reinforcement by different methods. In some cases, for example when carbon or SiC particles are used as reinforcement, detrimental products arising from chemical reactions at the metal-ceramic interface, such as aluminum carbide, $\mathrm{Al}_{4} \mathrm{C}_{3}$, are formed [11]. These products degrade the reinforcement at the interface and are, hence, undesirable to attain optimal mechanical properties. The occurrence of damage processes at the metal-ceramic interface is a crucial factor in the creep behavior. On the other hand, PM procedures avoid these reactions minimizing damage phenomena. Instead, a dispersion of $\mathrm{Al}_{2} \mathrm{O}_{3}$ particles of nano-meter size is unavoidably formed. This dispersion is, furthermore, beneficial for the creep behavior, and its effect should be also considered together with the specific role played by the ceramic particles of the micro-meter scale, intentionally introduced for composite fabrication [12]. Although these microstructural features derived from the processing route employed are also well known, the distinct role played by these two types of reinforcements (at the micro- and nano- scales) co-existing in the metallic matrix of PM MMCs, has been barely treated.

c) Most of the creep studies of MMCs do not compare the composite creep behavior with that of the un-reinforced alloy. Creep data comparison with that of the reference alloy is, however, essential to know the experimental composites creep strength enhancement. In fact, sometimes the addition of the reinforcement is detrimental to, rather than beneficial for, this behavior $[10,13]$. Composites creep data analysis without considering the behavior of the unreinforced reference alloy is, consequently, incomplete. For example, as above stated, one could be analyzing reinforcing mechanisms when, instead, other processes and/or severe damage are occurring during composite deformation resulting, even, in an inferior creep response than that of the un-reinforced alloy. Comparison of un-reinforced and composite creep data is, furthermore, not always a straightforward operation. For the case of the age-hardenable alloys, the accelerated ageing process which occurs in the composites [14] is an indication that the microstructure of the un-reinforced alloy and the composite matrix is not equivalent under specific given testing conditions. This factor should be also taken also into consideration when creep data comparison is conducted. 
In summary, a complex picture of the creep behavior of MMCs has resulted and a solid and accepted procedure to understand their response is still missing. Rather, the above factors have contributed to maintain during decades a confusing panorama on the understanding of this behavior.

Previous work by these authors on PM 6061Al-15vol\% $\mathrm{SiC}_{\mathrm{w}}$ MMC [15,16] have shown separately the role of the relevant microstructural factors affecting the enhanced composites creep behavior, namely, the dispersion of the aluminum oxide particles (nano-scale) introduced when the PM route is the one employed for materials preparation [15] and the ceramic particles (micro-scale), purposely introduced for enhancing mechanical and also creep properties [16]. Furthermore, a microstructural factor associated to the shorter inter-obstacle distance for dislocation motion should be also considered. In the present study, a simple and generalized picture of the relevant mechanisms required to understand the high temperature behavior of aluminum alloy metal matrix composites is proposed. Within this framework, the approach of the threshold stress associated to the ceramic particles, usually invoked to account for the creep of MMCs, will be criticized, and the additivity of the two main proposed mechanisms will be discussed. Besides previous work conducted by these author, the analysis derived from data of other investigations on the creep of MMCs will be also taken into account. As will be seen, a fundamental aspect of the present investigation is the need of comparing the creep behavior of the composite with respect that of the corresponding un-reinforced alloy which, as mentioned, has not been always taken into consideration.

\section{2.- The present situation: the threshold stress concept}

In spite of the above confusing picture, it is well accepted that the creep of discontinuously reinforced aluminum alloys MMCs must be understood in terms of the creep behavior of the metallic matrix alloy (only this phase undergoes plastic deformation due to the stiffer and stronger nature of the ceramic reinforcement). Therefore, knowledge and understanding of the creep behavior of the matrix alloys is the starting point to tackle the creep behavior of the composite. The creep of monolithic alloys is usually described by the power law creep equation in which the steady state creep rate, $\dot{\varepsilon}_{s s}$, is given by:

$$
\dot{\varepsilon}_{s s}=A^{\prime}\left(\frac{\sigma}{E}\right)^{n} \exp \left(-\frac{Q_{c}}{R T}\right)
$$

where $A^{\prime}$ is a material's microstructure constant, $E$ the Young's modulus, $n$ the stress exponent, $R$ the universal gas constant $(R=8.314 \mathrm{~kJ} / \mathrm{mol} \mathrm{K}), \sigma$ the applied stress, and $T$ the absolute 
temperature. On this base, one of the most significant consequences within the complex picture above mentioned is the consideration in several investigations (see for example, refs. [17-20]) of a threshold stress term, $\sigma_{0}$, associated to the ceramic particles as an additive contribution to the applied stress, but independent of it, into creep equation (1) such that the following equation should be obeyed.

$$
\dot{\varepsilon}_{s s}=A^{\prime}\left(\frac{\sigma-\sigma_{o}}{E}\right)^{n} \exp \left(-\frac{Q_{c}}{R T}\right)
$$

Equation (2) describes, then, the composite's matrix creep behavior. I.e., the composite's behavior after the strengthening effect of the reinforcement has been eliminated.

The term $\sigma_{\mathrm{o}}$ was proposed originally to understand the creep of oxide dispersion strengthened (ODS) alloys [21]. It was, then, firstly linked to the dispersion of the oxide particles of these materials. These ODS alloys show remarkably high values of the apparent $n$ and $Q_{\mathrm{c}}$. If sufficient data in the low applied stress regime are registered, the minimum or steady state creep rate decreases rapidly with the applied stress such that the apparent stress exponent, $n$, increases progressively deviating from the power law behavior, creep equation (1), as schematically illustrated in the double logarithmic representation of stress vs. strain rate of Figure 1. In other words, the stress-strain rate behavior reveals a curvature in a way that below a given stress (the threshold stress) no creep occurs in the material (the strain rate becomes immeasurably low). The incorporation of the threshold stress term in equation (2) "solves" this problem since "normal" $n$ (and $Q_{\mathrm{c}}$ ) values such as those of for monolithic alloys or pure metals, as schematically shown in the plot of Figure 1, are obtained.

The usual procedure to determine this threshold stress term is described elsewhere. In summary, it consists of "imposing" an $n$ value to the creep equation so that, at a given temperature, a straight line should be obtained when the $1 / n$ power of $\dot{\varepsilon}$ is plotted as a function of $\sigma / \mathrm{E}$. The extrapolated value of $\sigma$ at $\dot{\varepsilon}=0$ is taken as $\sigma_{0}$. The fits to obtain $\sigma_{\mathrm{o}}$ are made using typical $n$ values of $n=3, n=5$, and $n=8$, which are identified with specific deformation mechanisms [22]. As done in [12], the $n$ value which best fits a straight line and the corresponding $\sigma_{\mathrm{o}}$ are the data included in the creep equation. Figure 1 shows, schematically, the ODS alloy creep behavior in a double logarithmic representation before and after threshold stress normalization at a given temperature of testing. As can be seen, the "threshold behavior" manifested by the ODS alloy, and by definition a stress independent factor, is eliminated after this term is subtracted from the applied stress in the creep equation. In this manner, as described in equation (2), the creep 
behavior is "rationalized" to that of the matrix alloy as long as the incorporation of this term "eliminates" the reinforcing effect of the dispersion of oxide particles.

Surprisingly, a number of investigations have shown that, using the same method, a reasonable similarity between the creep behavior of MMCs and monolithic alloys or pure metals (in terms of stress exponent and activation energy), is obtained [17,23]. This encouraged many researchers to analyze the creep of MMCs in the framework of the presence of $\sigma_{\mathrm{o}}$ and to investigate the possible origins of this stress term. However, the different attempts to explain it from microstructural basis have, so far, failed. It is remarkable that very recent investigations do not even discuss the possible nature and/or origin of the threshold stress term.

To our understanding, this is a cul-de-sac situation which has prompted us to consider other possible ways to explain the creep of MMCs. For this purpose, it is first necessary to neglect resolutely the threshold stress term associated to the ceramic reinforcement. Several other reasons can be also invoked against this term despite the reasonable kinetics values obtained after threshold stress normalization to explain the creep of MMCs. These are:

1.- As above mentioned, little efforts have been focused in real data comparison between composite and un-reinforced alloy, and when this exercise is made no such expected agreement is found after threshold stress normalization of composite creep data [24]. To explain the discrepancy, further considerations, such as a load partitioning phenomenon, are also invoked, complicating further a general view of the creep behavior of MMCs.

2.- It has been also pointed out the limited reliability of the classical procedure to determine $\sigma_{\mathrm{o}}$ [14], in particular for the case of composites in which the matrix alloy is age hardenable [3,15,20,24-32]. When such alloys are used, $n$ is affected by the simultaneous precipitation process which occurs during creep. Therefore, the significance of the $\sigma_{o}$ values obtained by this method in these materials should be, at least, carefully considered.

3.- Usually, a strong variation with temperature of the threshold stress term has been found, with no explanation for it. The variations found are, generally, much higher that that expected from the Young modulus variation with temperature.

4.- The threshold stress like behavior, i.e., the curvature of the $\log \varepsilon-\log \sigma$ data in the low stress regime is not always evident.

5.- Contrarily to what it occurs in ODS alloys, for which the size of the particles is of the order of the dislocation core, the scale of the reinforcing particles (particle size and inter-particle distance) in MMCs is much larger (at least three orders of magnitude). Hence, the applicability 
of models describing the interaction of individual dislocations with particles to explain $\sigma_{\mathrm{o}}$ in MMCs is also doubtful [33].

At this point, it is important to emphasize that the rejection of $\sigma_{\mathrm{o}}$ associated to the presence of the ceramic particles does not disqualify the need of such stress term as linked to the dispersion of the aluminum oxide particles (when these composite materials are processed by a powder metallurgical route).

Recently, the importance of a partitioning phenomenon, typically active at room temperature, has been demonstrated to be also relevant at elevated temperatures [16], regardless that possible relaxation processes of the load transferred to the reinforcement may occur [34]. The importance of load transfer mechanism was also suggested in previous works $[13,24,26]$, and supported by an analysis of the creep data of composites and their respective un-reinforced alloys recorded from the literature $[16,35]$.

\section{3.- Mechanisms contributing to creep strength of aluminum alloy MMCs}

In the present work, we propose to extend and generalize the conclusions of this previous research on IM and PM 6061 Al alloys and a PM 6061 Al composite reinforced by $15 \mathrm{vol} \%$ of SiC particles $[15,16]$. From these investigations it is envisaged the importance of the metallurgical procedure in the possible contributing mechanisms to the enhanced composite creep behavior. The matrix alloy used in $[15,16]$ was $6061 \mathrm{Al}$, a typical alloy used in room temperature applications, but the conclusions derived, supported by the review data analysis conducted in [35], may be well extended to other aluminum alloys. It is proposed, hence, that the superior creep behavior of MMCs is explained on the basis of two main contributions, namely: a load transfer associated to the ceramic reinforcement and a stress independent threshold stress term associated to the oxide particles (which are present in the case of PM composites). Whereas predictive models to account for the relevance of each of these mechanisms can be used (Eshelby and/or Shear-Lag for the case of load transfer and the Artz model for the case of the threshold stress), the possible role of damage processes occurring at the interface is still difficult to predict. As described in [16], these mechanisms may be important in IM composites in which detrimental chemical reactions at the metal-ceramic interfaces are likely to occur.

A summary of all these mechanisms is presented in Table I. In this table, the fundamental contributing microstructural features derived from the processing route, the corresponding reinforcing mechanism, and the resulting creep equation are summarized for the un-reinforced alloys and their respective composite materials. 
Furthermore, a microstructural strengthening factor related to the change in the microstructure of the matrix aluminum alloy due to the addition of the reinforcement should be considered. The fact that the matrix alloy used (6061 Al) in the composite investigated in [16] is age-hardenable (and, therefore, that simultaneous precipitation during creep deformation occurs) should be also taken into account. In references $[15,16]$ the specific role played by this phenomenon as well as the increased dislocation density in the composite with respect the un-reinforced alloy (and the accompanying accelerated ageing process) has been analyzed in detail and will not be treated here. It should be bear in mind, however, that for other aluminum alloys, the addition of the reinforcement may cause (or not) a different change in the matrix microstructure and, hence, a different microstructural strengthening: different alloy matrix justify a different treatment on the effect of the reinforcement addition on the microstructure. The analysis of the specific role due to the microstructure change is not the objective of the present investigation and will be the subject of a separate work.

A as can be seen, the creep equation shown in Table I for the simplest case of a given unreinforced aluminum alloy obtained by IM is the conventional steady state creep equation (which has been taken in [15] as that which obeys the Sherby's sub-structure invariant model, with $n=8$ ). No contribution of the ceramic reinforcement and the dispersion of the oxide particles are, of course, taken into consideration. Also, damage mechanisms associate to metal-ceramic interface are absent. Depending of the aluminum alloy to be considered, the stress exponent and the steady state creep equation may differ from $n=3$ for the case of dislocation glide control behavior to $n=8$ for the case of creep under sub-structure invariant conditions.

For the case in which a PM route is involved in alloy preparation the creep equation should include a threshold stress term associate to the oxide alumina particles. For these alloys the creep rate is that described by equation (2). The $\sigma_{0}$ term can be calculated by the classical method, but it is convenient, however, to asses the value obtained with that resulting from data comparison of IM and PM alloy. It has been shown that the dislocation-particle interaction accounting for $\sigma_{\mathrm{o}}$ is well described by the Artz model [15,36,37]. A different microstructural factor $A$ in equations (1) and (2) should be also taken into consideration to determine $\sigma_{\mathrm{o}}$ experimentally [16]. Again, damage mechanisms associate to the interface are absent.

When the ceramic particles are purposely embedded into the aluminum alloy matrix by an IM procedure to obtain a composite, the "unique" reinforcing mechanisms that should be in principle considered is the load transfer mechanisms. In table I, the associate equation to describe this phenomenon is that derived from the Shear-Lag model proposed by Ryu [38], but, depending on 
the reinforcement morphology, other Shear-Lag or Eshelby model may be appropriate as well [16]. Here, the commonly used threshold stress term considered in several investigations, equation (2), is simply replaced by the load partition factor which can be obtained experimentally from composite and un-reinforced alloy creep data comparison [16]. I.e., the mathematical description of the composite creep equation is similar to that commonly used with the $\sigma_{\mathrm{o}}$ term, but replacing $\sigma_{\mathrm{o}}$ by $\sigma_{\mathrm{T}}$ in equation (2). Additional strengthening (or weakening) may be achieved as a consequence of microstrutural refinement (Constant $A$ in the composite may differ from that of the un-reinforced alloy as a consequence of reinforcement addition). Potential damage phenomena, resulting in diminishing mechanical and creep properties, should be also borne in mind in this type of composites. Quantification of these phenomena is, however, difficult, but can be of major importance in these materials. Finally, relaxation of load transferred to the reinforcement may be also of certain importance $[13,34]$.

In the final case in which the reinforcing ceramic particles are introduced by a powder metallurgical route, both the load partitioning phenomenon as well as the threshold stress should be considered for the improved creep behavior. Again, the models above discussed (Ryu's [38] and Artz's [36,37] for the load transfer effect and threshold stress, respectively), or other equivalent models, can be employed in the mathematical treatment of these two contributions. In PM composites, the unlikely formation of detrimental chemical reactions at the metal-ceramic interface indicates that damage phenomena associate to this interface should be minimized. The additivity of these two contributions to the strengthening of the PM composites will be discussed in the following section. As in the IM composites, the load transferred relaxation process may be also important in a mathematical treatment of the composite creep behavior.

\section{4.- Additivity of reinforcement contributions}

In case of the PM composite material, both reinforcing mechanisms coexist during creep, Table I. However, the origin of each contribution is different. At the beginning of the creep test, when the external load is applied to the material, this is part of this stress is transferred elastically to the micrometric reinforcement. Therefore, there is a fraction of the load borne by the micrometric reinforcement from the first moment of the test, $\sigma_{T}$. Thus, the effective stress for dislocation motion during creep of the composite is $\left(\sigma-\sigma_{T}\right)$. This transferred stress has been calculated by Shear-Lag and Eshelby methods and fit quite successfully with the experimental values obtained from creep data comparison of un-reinforced alloy and composite material [16]. Besides this decrease in the effective stress for creep deformation, dislocation motion it further inhibited by the presence of the oxide dispersion in the same manner as done for the PM alloys. 
Part of the remaining stress $\left(\sigma-\sigma_{T}\right)$ resulting from the load partition process is needed for the dislocation detachment process from the alumina dispersoids: i.e., a stress term explained through the so called threshold stress, $\sigma_{0}$, and assessed by the Artz's model [36,37]. The different origin of both reinforcing contributions allows, then, treating these terms in an additive manner. It is finally worth mentioning the different effect of these two reinforcing mechanisms on the power law creep behaviour of the composite and the resulting apparent stress exponent. Whereas the load transfer mechanism does not affect $n$ (the load transferred increases with the applied stress) the $\sigma_{\mathrm{o}}$ term does, as it is, by definition, stress independent. This is also in agreement with the model equations accounting for these two mechanisms: Ryu's [38] and Artz's [36,37], for the load transfer mechanism and the threshold stress, respectively, as depicted in Table I. This is also seen from the plot of Figure 2 where experimental creep data of 6061 Al alloy, obtained by both $\mathrm{IM}$ and $\mathrm{PM}$ procedures, as well as data of $6061 \mathrm{Al}-15 \mathrm{vol} \% \mathrm{SiC}_{\mathrm{w}}$ composite at $673 \mathrm{~K}$ are represented $[15,16]$. As can be seen, $n$ is lower in the IM alloy, and is due to the presence of a threshold stress, $\sigma_{\mathrm{o}}$, in the PM materials, absent in the IM alloy. The increased creep behaviour of the PM composite with respect the PM alloy is due to the load transfer effect. Also, the expected similarity between their high apparent stress exponents is because the $\sigma_{\mathrm{o}}$ term affects the creep behaviour of both materials in the same way.

\section{5.- Summary}

In this work, a simple and generalized picture of the relevant mechanisms required to understand and predict the creep behavior of discontinuously reinforced metal matrix composites, particularly aluminum alloy matrix materials, is proposed. The investigation was motivated due to the present confusing situation with respect the different views to understand the creep of these types of materials. Based on previous work on the creep of PM 6061Al matrix composite and respective PM and IM un-reinforced alloys and a review data analysis from other published investigations, we were encouraged to generalize the conclusions derived to different aluminum alloys matrix composites.

In particular, the threshold stress term associated to the ceramic reinforcement of micrometer scale is rejected. It is proposed, instead, the relevance of a load transfer mechanism as the reinforcing mechanisms associated to these particles. The presence of a threshold stress, independent of the applied stress, is, hence, released to the dispersion of aluminum oxide particles, of nanometer scale, in the case of composites (and alloys) prepared by PM procedures. These particles are inevitably formed in these materials by PM procedures. 
The possible creep strengthening contributions and the potential damage mechanism at the metal-ceramic interface are, hence, discussed in the context of the processing route employed for composites preparation. In particular, emphasis is made on the additive contribution of the two main reinforcing mechanisms that should be considered in materials processed by a PM route: a load partitioning phenomenon and a threshold stress, associated to the ceramic reinforcement and the aluminum oxide particles dispersion, respectively.

\section{Acknowledgements}

Project MAT05-00527 from MEC, Spain. 
$07 / 07 / 2008$

\section{References}

1.- A.L. Geiger, J.A.Walker, JOM 45 (8) (1991) 8-15.

2.- M.K. Aghajan, C.A. Anderson, R.J. Wiener, B.R. Rossing, SAE Int Congress and exp.(1995).

Detroit, Michigan. SAE Technical Paper Series. p.1-7.

3.- T.G. Nieh, Metall. Trans. A15 (1984) 139-145.

4.- T.W. Clyne, P.J. Whiters, An introduction to metal matrix composites, Cambridge University Press, Cambridge,1993: p.459.

5.- A. Borrego, G. González-Doncel, Mater. Sci. Eng. A245 (1998) 10-18.

6.- C. García-Cordovilla, E. Louis, J. Narciso, A. Pamies, Mater. Sci. Eng. A189 (1994) 219227.

7.- J.M. Papazian, Metall. Trans. 19A (1988) 2945-2953.

8.- M.H. Jacobs, Philos. Mag. 26 (1972) 1-13.

9.- Y Song, T.N. Baker, Mater. Sci. Eng. A201 (1995) 251-260.

10.- G.C. Requena, H.P. Deguischer, Z. Metallkd. 96 (2005) 805-811.

11.- D.J. Lloyd, Comp. Sci. Technol. 35 (1989) 159-179.

12.- K.T. Park, E.J. Lavernia, F.A. Mohamed, Acta Metall. Mater. 42 (1994) 667-678.

13.- P.E. Krajewski, J.E. Allison, J.W. Jones, Metall. Trans. 24A (1993) 2731-2741.

14.- A. Borrego, J. Ibáñez, V. López, M. Lieblich, G. González-Doncel, Scripta Mater. 34 (1996) $471-478$.

15.- R. Fernández, G. González-Doncel, J. Alloys and Comp. 440 (2007) 158-167.

16.- R. Fernández, G. González-Doncel, Acta Mater. 56 (2008) 2549-2562.

17.- G. González-Doncel, O.D.Sherby, Acta Metall.Mater. 41 (1993) 2797-2805.

18.- Y. Li, F.A. Mohamed, Acta Mater. 45 (1997) 4775-4785.

19.- Y. Li, T.G. Langdon, Scripta Mater. 36 (1997) 1457-1460.

20.- S. Spigarelli, M. Cabibbo, E. Evangelista,T.G. Langdon, Mater. Sci. Eng. A328 (2002) 3947.

21.- J. Lin, O.D. Sherby, Res Mecánica 2 (1981) 251-293.

22.- O.D.Sherby, B. Walter, Scripta Metall. 16 (1982) 213-219.

23.- R.S. Mishra, A.B. Pandey, Metall. Trans. 21A (1990) 2089-2090.

24.- K.T. Park, F.A. Mohamed, Metall. Mater. Trans. 26A (1995) 3119-3129.

25.- Y. Li, T.G. Langdon, Metall. Mater. Trans. 29A (1998) 2523-2531.

26.- H.J. Ryu, H. Chung, I.C. Seung, S.H.Hong, J. Mater. Res. 19 (2004) 3633-3640. 
27.- J.R. Pickens, T.J. Langan, R.O. England, M. Liebson, Metall. Trans. 18A (1987) 303-312.

28.- J. Komenda, P.J. Henderson, in: Proceedings of the 12th Riso International Symposium, (1991) 449-454.

29.- D. Yu, T. Chandra, Mater. Sci. Forum 113-115 (1993) 563-568.

30.- M. Furukawa, J. Wang, Z. Horita, M. Nemoto, Y. Ma, T.G. Langdon, Metall. Mater. Trans. 26A (1995) 633-639.

31.- N. Matsuda, J. Akaike, K. Hongo, K. Matsuura, Mater. Sci. Eng. A234 (1997) 751-754.

32.- K. Wakashima, T. Moriyama, T. Mori, Acta Metall. Mater. 48 (2000) 891-901.

33.- A. Yawny, G. Eggeler, Mater. Sci. Eng. A387-389 (2004) 905-909.

34.- K. Kucharova, J. Cadek, S.J. Zhu, J. Mater. Sci. 38(2003) 3535-3543.

35.- R. Fernández, G. González-Doncel, submitted to Mater. Sci. Eng. A.

36.- E. Artz, S.D. Wilkinson, Acta Metall. 34 (1986) 1893-1898.

37.- J. Rösler, E. Artz, Acta Metall. Mater. 38 (1990) 671-683.

38.- H.J. Ryu, S.I. Cha, S.H. Hong. J. Mater. Res. 18 (2003) 2851-2858. 


\begin{tabular}{|c|c|c|c|c|c|}
\hline Material/Processing Route & Load transfer; $\sigma_{\mathrm{T}}$ & Threshold stress; $\sigma_{\mathrm{o}}$ & Stress exponent & Creep equation*. & Interface damage \\
\hline un-reinforced alloy/IM & NO & NO & $n_{\mathrm{IM}} \in[3-8]$ & $\dot{\varepsilon}_{s s}=A\left(\frac{\sigma}{E}\right)^{n} \exp \left(-\frac{Q_{c}}{R T}\right)$ & NO \\
\hline un-reinforced alloy/PM & $\mathrm{NO}$ & $\sigma_{o}=0.84 \frac{G b}{L-d} \sqrt{1-k^{2}}$ & $n_{\mathrm{PM}} \geq n_{\mathrm{IM}}$ & $\dot{\varepsilon_{s s}}=A\left(\frac{\sigma-\sigma_{D}}{E}\right)^{n} \exp \left(-Q_{c} / R T\right)$ & NO \\
\hline composite/IM & $\sigma_{T}=\sigma\left(\frac{f\left(S_{e f f} / 2+1\right)}{f\left(S_{e f f} / 2+1\right)+(1-f)}\right)$ & NO & $n_{\mathrm{IM}}$ & $\dot{\varepsilon}_{s s}=A\left(\frac{\sigma-\sigma_{T}}{E}\right)^{n} \exp \left(-Q_{c} / R T\right)$ & major \\
\hline composite/PM & $\sigma_{T}=\sigma\left(\frac{f\left(S_{S f f} / 2+1\right)}{f\left(S_{S f f} / 2+1\right)+(1-f)}\right)$ & $\sigma_{o}=0.84 \frac{G b}{L-d} \sqrt{1-k^{2}}$ & $n_{\mathrm{PM}} \geq n_{\mathrm{IM}}$ & $\dot{\varepsilon}_{s s}=A\left(\frac{\sigma-\sigma_{T}-\sigma_{0}}{E}\right)^{n} \exp \left(-Q_{c} / R T\right)$ & minor \\
\hline
\end{tabular}

* Constant $A$ in the different equations was taken as the same one, but it may differ from un-reinforced alloy to the composite depending of the processing route and effect of reinforcement.

Table I. Summary of the fundamental strengthening contributions proposed for the enhanced creep behavior of discontinuously reinforced MMCs (and corresponding un-reinforced alloys) associated to the presence of each type of reinforcement. Their mathematical description according to specific model, the corresponding stress exponent values, the creep equation, and the potentiality of damage associated to the metal-ceramic interface are also included. 


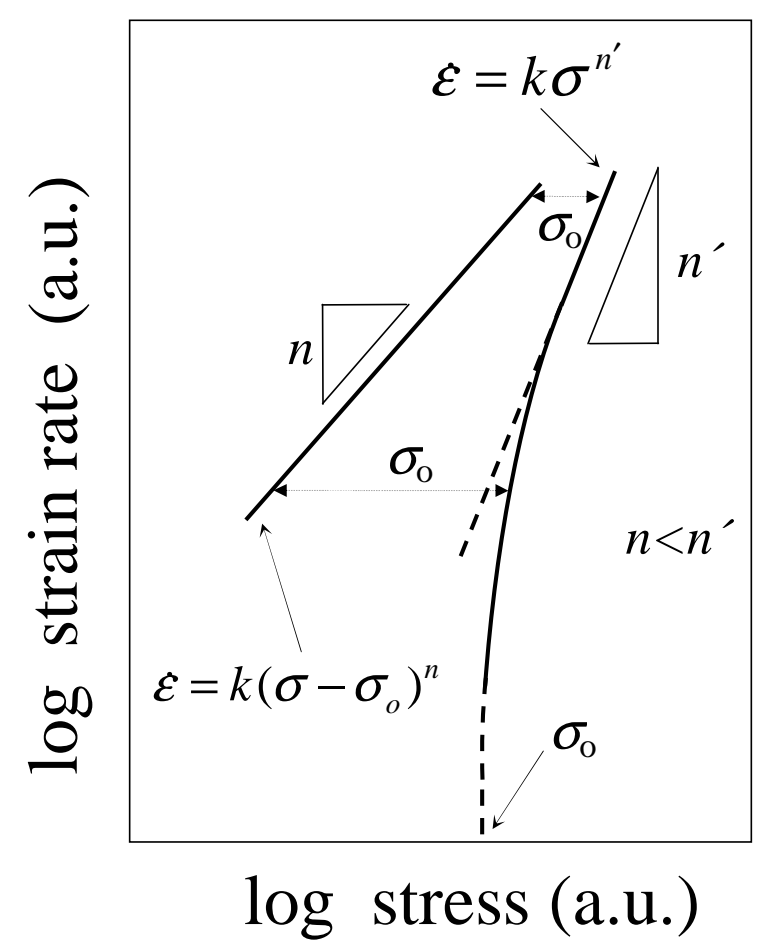

Figure 1.- Schematic representation of the "threshold stress" like behavior in a double logarithmic representation of the stress vs. strain rate. As seen, this behavior is eliminated after the incorporation of the $\sigma_{0}$ term in the exponential creep equation. 
$07 / 07 / 2008$

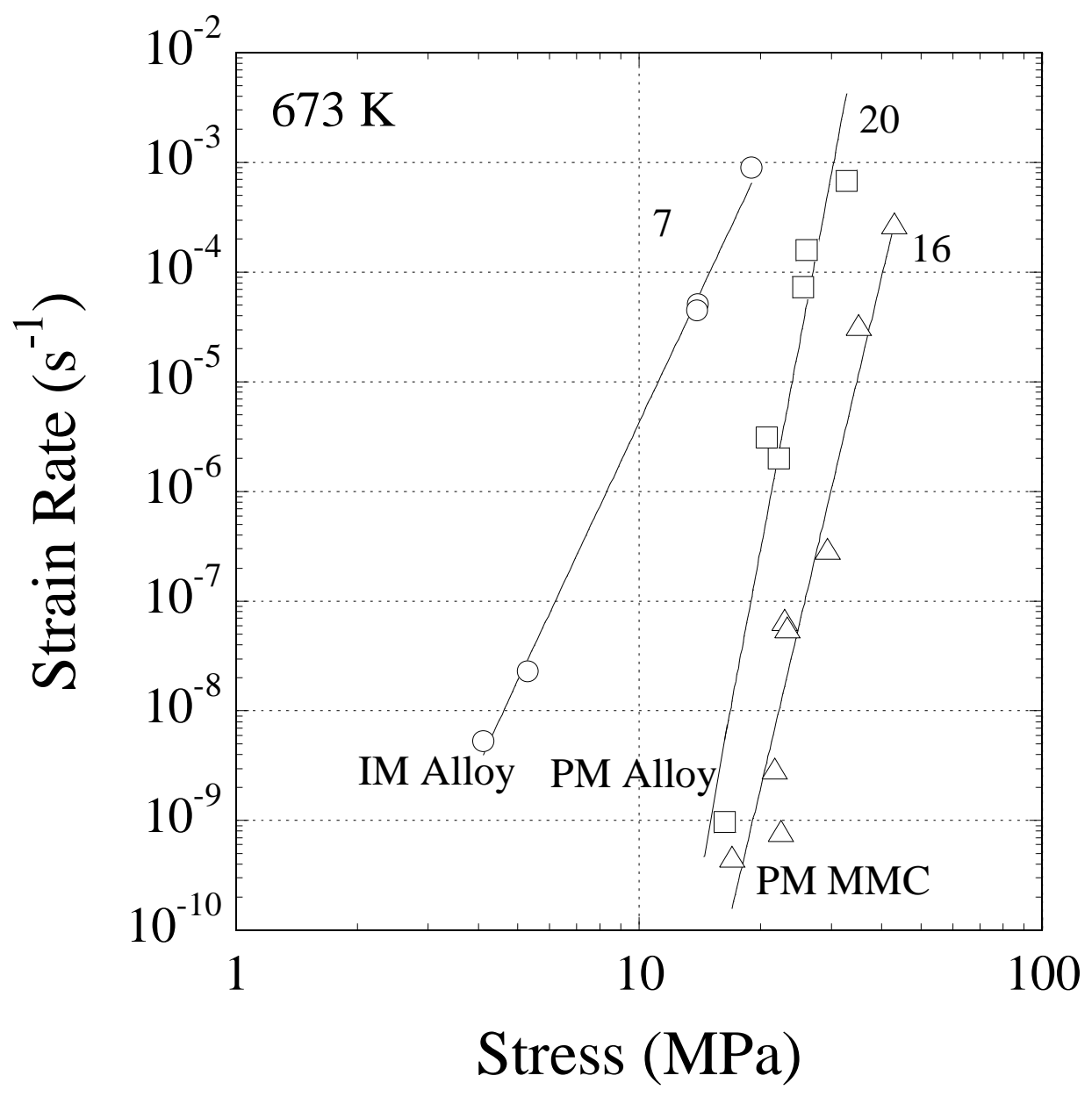

Figure 2.- Strain rate as a function of the stress (in $\log -\log$ scale) at $673 \mathrm{~K}$ of a $6061 \mathrm{Al}$ alloy obtained by IM (IM Alloy) and PM (PM Alloy) and a PM 6061Al-15vol\% SiC $_{\mathrm{w}}$ composite (PM MMC). The apparent stress exponent is similar for the PM materials and higher than that of the IM alloy. Data obtained form refs. $[15,16]$. Numbers denote the stress exponent. 


\section{Figures captions}

Figure 1.- Schematic representation of the "threshold stress" like behavior in a double logarithmic representation of the stress $v s$. strain rate. As seen, this behavior is eliminated after the incorporation of the $\sigma_{\mathrm{o}}$ term in the exponential creep equation.

Figure 2.- Strain rate as a function of the stress (in log-log scale) at $673 \mathrm{~K}$ of a $6061 \mathrm{Al}$ alloy obtained by IM (IM Alloy) and PM (PM Alloy) and a PM 6061Al-15vol\% $\mathrm{SiC}_{\mathrm{w}}$ composite (PM MMC). The apparent stress exponent is similar for the PM materials and higher than that of the IM alloy. Data obtained form refs. $[15,16]$. Numbers in parenthesis denote the stress exponent. 